\title{
Dinámicas recientes de simplificación administrativa y racionalización de trámites en Colombia frente al contexto internacional
}

Fecha de recepción: 15 de enero de 2011

Fecha de aceptación: 22 de marzo de 2011

\author{
Naidú Duque Cante \\ naduquec@poli.edu.co \\ Politécnico Grancolombiano
}

Administradora Pública y magíster en Administración Pública de la Escuela Superior de Administración Pública ESAP. Con experiencia administrativa, investigativa y docente. Miembro del grupo de investigación "Descentralización y Gestión Territorial" de la ESAP desde el año 2003, reconocido por Colciencias. Docente de planta de tiempo completo del Pregrado en Administración Pública del Politécnico Grancolombiano en las materias de Pensamiento Administrativo Público, Organización del Estado y Administración Local y Desarrollo Municipal. Docente de cátedra de Relaciones Intergubernamentales en Colombia, Trabajo de Grado y Metodología de la Investigación de la facultad de Administración Pública de la ESAP. Experta en organización del Estado, descentralización territorial en Colombia y "descentralización" y organización interna en Bogotá.

\section{Resumen}

Con el propósito de analizar los avances que ha realizado nuestro país en materia de simplificación y racionalización en administración pública, especialmente en lo correspondiente a los trámites que deben llevar a cabo particulares y empresas frente al Estado, este documento recoge los desarrollos académicos y normativos que han rodeado la consolidación de las bases institucionales alrededor del tema tanto en Colombia como en el contexto internacional.

A partir de este análisis fue posible identificar algunos elementos de gran importancia que se encuentran presentes en los desarrollos normativos de los países tomados como referencia. Dichos desarrollos evidencian que Colombia todavía adolece, en su marco institucional, de énfasis importantes vinculados con el uso de tecnologías de la información y con instrumentos de protección e

\begin{abstract}
In order to analyze the progress made by our country in terms of simplification and rationalization of public administration, especially for the formalities to be carried out by individuals and companies against the State, this document contains academic and policy developments that have surrounded the consolidation of institutional bases around the topic, not only in Colombia, but also within the international context.

From this analysis it was possible to identify some important elements that are present in the regulatory developments of the countries taken as reference. These developments show that Colombia still suffers, within its institutional framework, significant emphasis related to the use of information technology and instruments for the protection and dialogue with society. The importance of these elements is the usefulness they provide to
\end{abstract}


interlocución con la sociedad. La importancia de estos elementos radica en la utilidad que prestan tanto a la sociedad como al Estado, por un lado en el mejoramiento de los niveles de satisfacción y por otro, en la calidad de los servicios que ofrecen los distintos organismos y entidades del sector público.

\section{Palabras clave}

Simplificación, trámites, e-gobierno, tecnologías de la información y la comunicación. both the society and the State. First in improving satisfaction levels, and secondly, in improving the quality of the services offered by different agencies and public sector institutions.

\section{Keywords}

Simplification, Procedures, e-government, Information and Communication Technologies. 


\section{Introducción}

Las tendencias recientes de transformación del Estado se han caracterizado por el énfasis reduccionista del tamaño y el número de organismos de carácter público, además de la aparición de un nuevo paradigma teórico: la Nueva Gestión pública. Este paradigma ha buscado adaptar la administración pública a las nuevas condiciones que le exige el contexto al aparato estatal mediante la adopción de valores y criterios de carácter privado en el funcionamiento del sector público. Entre los más importantes encontramos la eficiencia, la eficacia y la orientación al usuario, los cuales han derivado en el replanteamiento de las relaciones entre el Estado y la sociedad y a la conversión del ciudadano en cliente.

Todo este marco institucional ha conducido, entre otros resultados, al desarrollo de los actuales procesos de simplificación y racionalización administrativa tanto para nuestro país como para el contexto internacional. Adicionalmente, las medidas de racionalización se han combinado con la adopción, asimilación y difusión permanente de las tecnologías de la información dentro de la tendencia del e-gobierno o gobierno electrónico.

Con el ánimo de comprender cuáles han sido los avances de nuestro país en materia de simplificación administrativa y racionalización de trámites, este documento se construyó tomando como referencia no sólo los desarrollos académicos alrededor del debate teórico de la temática, sino además los avances en materia normativa de nuestro país frente a las dinámicas de países como España, Francia, Canadá, Chile y México.

Adicionalmente, se tomaron en cuenta los informes oficiales y académicos sobre los desarrollos en relación con las tecnologías de la información y los aportes que dichas tecnologías han hecho al mejoramiento de las relaciones entre el Estado y la sociedad en materia de trámites. El análisis se fundó en la comparación para los países mencionados, de los aspectos vinculados con los contenidos de las diferentes políticas para el mejoramiento de los vínculos de la administración con los particulares, el mejoramiento de procesos internos y la asimilación de las tecnologías en la implementación de nuevos procesos y procedimientos de trámites.

\section{De la transformación del Estado al mejoramiento de las relaciones con la sociedad}

Entre las explicaciones más importantes que fundamentan las últimas dinámicas de transformación del Estado en América Latina, es posible destacar, como causas originarias, por un lado, la crisis fiscal soportada en el crecimiento exponencial de la deuda externa. Dicha situación se justificó, en su momento, por el crecimiento desbordado del tamaño del Estado debido al incremento de funciones en sus dinámicas de intervencionismo económico y de benefactor social.

Por otro lado y quizás en un sentido distinto, hubo una segunda manifestación de la crisis, que se evidenció en una excesiva centralización de los procesos de toma de decisiones y de asignación del gasto público, originando la denominada crisis del centralismo. Dicha crisis en particular se debió a que el centralismo 
impedía que las decisiones se ajustaran a las necesidades, preferencias y problemáticas reales de la sociedad en el nivel local. De tal forma que, aunque el Estado demandaba muchos recursos fiscales, su asignación no conducía al mejoramiento de los niveles de bienestar deseables para la sociedad.

Ambas crisis dieron lugar a mutaciones distintas en la configuración de la institucionalidad formal que rige desde entonces el diseño del Estado, especialmente en América Latina. En términos de Moncayo (2006) el poder del Estado se desbordó en tres direcciones distintas. En primer lugar, hubo un desbordamiento del poder horizontalmente, debido al desmonte del Estado de bienestar inducido por el modelo neoliberal, que buscaba la predominancia del mercado y preconizaba la necesidad irrefutable de un "Estado mínimo". La consecuencia de este cambio se manifestó en los procesos de privatización generalizada que experimentaron los Estados latinoamericanos.

Esta transformación del Estado, en particular, estuvo justificada por los diagnósticos de los expertos neoliberales quienes consideraron que la crisis estaba estrechamente relacionada con el modelo de organización social fundado en una matriz estadocéntrica, la cual condujo a una intervención desmedida del Estado, a su sobredimensionamiento y al estímulo de una conducta clientelar y predatoria de los controladores del aparato público (Ramírez, 2009).

En segundo lugar, se produjo un desbordamiento del poder hacia abajo debido a los procesos de descentralización y entrega de autonomías a los niveles subnacionales del Estado, cuyo énfasis se tradujo en propósitos de fortalecimiento de la democracia a través de iniciativas de elección popular de autoridades políticas y de ejercicios participativos en los procesos de toma de decisiones. Asimismo, estas tendencias han buscado mejorar la gestión pública a nivel local, al garantizar un mejoramiento en la calidad de la planeación y focalización del gasto público.

En tercer lugar, el poder se desbordó hacia arriba por la globalización y la supranacionalización, debido al surgimiento de organismos e instituciones de carácter internacional y supranacional que han usurpado el poder de los Estados en diversos ámbitos. A este respecto, los Estados ya no manejan "autónomamente asuntos como el modelo de desarrollo, el sistema político, los derechos humanos, el medio ambiente, la justicia y la seguridad nacional" (Moncayo, 2006, p. 3).

Frente a la aparición de una nueva dinámica de configuración y funcionamiento estatal fue necesario, además, el desarrollo de un nuevo paradigma teórico, tendiente a la adecuación de la administración pública coherente con las lógicas del Estado neoliberal. Dicho paradigma, denominado Nueva Gestión Pública, abrió las puertas hacia una administración más consecuente con las nuevas funciones y perspectivas de desempeño que rodearon el sector público desde la implantación del nuevo modelo de desarrollo.

En particular, durante las denominadas reformas de segunda generación, que siguieron a aquellas que se fundaron en la filosofía del Estado mínimo, el nuevo paradigma administrativo público adquirió gran importancia dentro de los procesos de fortalecimiento institucional. El propósito de éstos, cuyo hilo conductor siguió siendo el modelo neoliberal, fue combatir los vicios burocráticos 
derivados de la aplicación del tradicional paradigma weberiano de administración pública característico de la era del Estado de bienestar. Las burocracias de este período se fundaron en la adopción de la lógica de la producción en serie proveniente del fordismo, buscando proveer masivamente servicios sociales universales a todos los habitantes del Estado. Esto generó la aparición de grandes organizaciones de carácter público encargadas de suministrar servicios como la educación, la salud, la seguridad alimentaria, el agua potable, los transportes masivos, la electricidad, los subsidios al desempleo, entre otros (Rodríguez y Tapia, 2008).

El funcionamiento de estas burocracias se caracterizó por la creciente expansión en el número y en la especialización de los funcionarios públicos, lo que derivó en la ampliación del tamaño de las nóminas de personal y de los sistemas de carrera, así como de los ámbitos de intervención. Al mismo tiempo, todo este aparato administrativo debió fundarse en una expansión creciente del gasto público y en el crecimiento exponencial de la deuda pública en todos los países de América Latina.

En este sentido, uno de los principales argumentos para introducir la doctrina neoliberal como la única alternativa frente a la crisis, se fundó en la ineficiencia del sector público y el excesivo gasto, en contraste con el menor crecimiento de los ingresos públicos, que a la larga no se veía reflejado en un mejoramiento de las condiciones sociales de las poblaciones ni en la reducción de las inequidades entre clases sociales y regiones. A esta situación se sumaron argumentos como la excesiva regulación estatal y el agotamiento del modelo desarrollista de sustitución de importaciones, así como la resistencia al cambio de los organismos y funcionamiento de los organismos públicos (Hernández, 2007).

Así, la búsqueda por conducir al Estado hacia mejores niveles de desempeño se fundó en un paradigma cuyos fundamentos partieron del éxito acumulado por el sector privado durante el siglo XX, lo cual implicó la asimilación en el ámbito público de las denominadas ciencias de la gestión o management.

Esta nueva tendencia teórica, aunque no cuenta con un cuerpo de conocimientos único ${ }^{1}$, se alimenta de una diversidad de planteamientos cuyo lugar de convergencia se ubica en el acuerdo alrededor de la introducción de una lógica empresarial en el sector público. Su propósito es conducir al Estado y su administración hacia un mejoramiento continuo en materia de provisión y garantía de bienes y servicios públicos, tomando como referentes los principios de eficiencia, eficacia, economía, calidad, transparencia y orientación al cliente, entre otros.

En estos términos, el nuevo paradigma ha buscado...orientar la prestación de servicios de la administración pública del ciudadano al consumidor, aumentar la eficiencia de los servicios desarrollando el mercado en su seno, sustituir el servicio civil de carrera por el de contrato, donde prevalezca la evaluación de resultados y los incentivos; $y$, someter las operaciones gubernamentales a la competencia interna y externa basándose en razones del mercado

De acuerdo con Isabel María García Sánchez (2007), entre las corrientes teóricas que contribuyen a la fundamentación teórica de la Nueva Gestión Pública están el Public Choice, los Costes de Transacción, el Neo-tailorismo y la Teoría de la Agencia. También se puede consultar a Lozada, 2008. 
(Guerrero, 1998, p. 28). En esta lógica, de acuerdo con López (2003), en la búsqueda del mejor funcionamiento del sector público, la etapa inicial de aplicación de las tecnologías de gestión privada en el ámbito de las organizaciones públicas, incluyó la racionalización de estructuras y procedimientos, la revisión de los procesos de toma de decisiones y el incremento de la productividad de los empleados públicos (2003, p. 7).

En síntesis, la OCDE (1995, p. 28) identificó como elementos de las reformas de la administración pública con énfasis empresarial los siguientes: 1) Devolver autoridad, otorgar flexibilidad, 2) Asegurar el desempeño, el control y la responsabilidad, 3) Desarrollar la competencia y la elección, 4) Proveer servicios adecuados y amigables a los ciudadanos, 5) Mejorar la gerencia de los recursos humanos, 6) Explotar la tecnología de la información, 7) Mejorar la calidad de la regulación, y 8) Fortalecer las funciones de gobierno en el centro.

Aunque esta propuesta recoge y sintetiza los instrumentos que identifican la tendencia de la Nueva Gestión Pública en lo que podría considerarse un modelo único, también considera que puede adaptarse de acuerdo con las necesidades y características del ámbito institucional del país respectivo. Esto supone la existencia de países con mayores grados de desarrollo en uno o varios de estos aspectos, exigiendo que su adaptación e incorporación sea distinta para cada ámbito particular.

\section{La simplificación administrativa dentro de las dinámicas de transformación del Estado}

Estas nuevas lógicas de transformación del Estado y su administración pública han conducido al replanteamiento de las relaciones entre éste y la sociedad, por un lado cuando se trata de los procesos de descentralización y la implementación de mecanismos de democratización de la vida local y, por otro, cuando se refiere a la transformación del ciudadano en "cliente". Esta última condición en la que se ha ubicado a los individuos que forman la sociedad se fundamenta en la necesidad de mejoramiento permanente de la calidad de los bienes y servicios públicos a partir de la consideración de las actitudes y los comportamientos del cliente-ciudadano, para satisfacer plenamente sus demandas y expectativas (Lawrence R. y Thompson, 1999, citados por López, 2003).

Lo anterior depende, además, de una mayor sensibilización de la administración pública respecto a los individuos y grupos a los que sirve (Lozada, 2008, 45). La satisfacción del cliente se convierte en un punto de referencia importante para medir la calidad del sector público dentro de la nueva lógica de calidad total que se importa desde el sector privado. Dicha prerrogativa plantea la necesidad de que los modelos políticos y administrativos deban construirse a partir del ciudadano y en función de sus necesidades colectivas, lo cual se desprende de la premisa de que el ciudadano es el dueño o propietario de la administración pública (Rodríguez, 2002).

En este contexto, la legitimación de la administración pública depende de los 
resultados, que están condicionados por la capacidad de acción del sector público para satisfacer las nuevas demandas sociales. Esto implica dar prioridad a la producción y entrega de bienes y servicios a los ciudadanos considerados como destinatarios, dentro de un marco legal y constitucional y teniendo como base que la función de la administración está justificada en una actuación objetiva (Rodríguez, 2002). En este sentido, como lo plantea Tornos (2000), se busca pasar de una administración legitimada por el cumplimiento de la norma a una administración legitimada por una actuación que maximice la satisfacción de los ciudadanos a los que sirve.

Esta perspectiva, sin embargo, riñe en gran medida con los propósitos de acercamiento del Estado al ciudadano, derivados de los procesos descentralistas y democratizadores. Desde la perspectiva de analistas como Richards (1994), la diferencia entre el ciudadano y el cliente se debe a que en el primer caso se considera la existencia de un interés colectivo que no es asimilable a la suma de los intereses individuales. Por su parte el cliente es apenas un consumidor cuyo móvil es el interés particular.

Otros análisis, sin embargo, buscan establecer una especie de híbrido que combine las dos lógicas propuestas en lo que se ha denominado el ciudadano-cliente, especialmente en referencia a la formulación y evaluación de políticas públicas. El traslado de una lógica fundada en la democracia representativa a una en la que se da predominancia a la democracia participativa, implica un mayor involucramiento de la opinión ciudadana en los procesos de diseño de las decisiones públicas. Asimismo, este nuevo panorama exige la medición en la satisfacción de la sociedad frente a la implementación de las decisiones y los procesos permanentes de rendición de cuentas desde los organismos del sector público. Dentro de la dinámica de evaluación, con el ánimo de medir la calidad del sector público, se utilizan los objetivos y valores de los usuarios como criterio para valorar la politica, negando un papel protagonista a los objetivos y metas de los responsables de ésta (Bañón y Carrillo, 1997).

Las características de organización y funcionamiento de la administración pública pueden depender de múltiples factores entre los cuales podemos contar la variación en la importancia relativa de los servicios, la cual se vincula con la transformación de las prioridades incluidas en la política del gobierno; los cambios en la composición de la población, a partir de los procesos migratorios, derivando en un mayor volumen y características de los servicios demandados y, finalmente, la creación de nuevas necesidades, provocada por la mayor concentración de población urbana y por la evolución natural de la sociedad por efectos de las tendencias globalizantes y la importación de preferencias y expectativas.

En esa perspectiva, el conjunto de elementos que marcan las tendencias reformadoras desde la configuración de la administración, aunque, en general, usen técnicas y herramientas provenientes del sector privado, deberán en mayor o menor medida consultar las exigencias y características propias de la sociedad y el contexto económico, tecnológico e institucional dentro de la cual funcionan. Adicionalmente, es necesario consultar la validez de cada una de las técnicas dentro de las dinámicas organizacionales del sector público. En este caso de acuerdo con Echebarría y Mendoza (1999, citados por López, 2003), se pueden establecer 
tres grupos distintos que permitirían clasificar estas técnicas en términos de: aplicación directa, adaptación creativa y necesidad de reconceptualización. En este último caso se trata de la reinvención de los instrumentos de gestión, con base en la especificidad del sector público.

Toda esta lógica de readaptación de los aparatos administrativos y la necesidad de establecer mejores lazos de relacionamiento entre el Estado, el ciudadano y el sector privado empresarial, plantea el imperativo de permanentes procesos de racionalización y simplificación administrativa que mejoren los canales y mecanismos de contacto público-privado. Lo anterior, con el fin de ajustar el funcionamiento del Estado a las disposiciones legales que por un lado obligan a los ciudadanos y a las empresas a establecer contacto con los despachos públicos y por otro, ofrecen garantías a la sociedad en materia de mejores condiciones de vida por el acceso a bienes y servicios de carácter colectivo.La racionalización de la administración pública busca, entre otros propósitos (Sánchez, 1993), una asignación precisa de funciones y la adecuación de las estructuras organizacionales a las mismas, la eliminación de duplicidades burocráticas y la reducción de conflictos paralizantes entre entidades públicas, con el propósito de dar una mayor coherencia al conjunto de la acción administrativa y una mayor reducción del gasto público.

Estos ajustes y adaptaciones propios de la administración pública requieren de procesos de racionalización administrativa de manera permanente, es decir, no se trata de un proceso único y definitivo. Esto, debido a que si no se traza un plan adecuado o no se realiza un seguimiento y control consecuentes con su ejecución y el cumplimiento de sus objetivos generales, las tendencias de ajuste pueden revertirse. A este respecto, las medidas iniciales de reducción del tamaño del Estado en aras de un Estado mínimo se vieron en un primer momento contrarrestadas por un incremento de la burocracia, medida en número de funcionarios y entidades públicas en los niveles subnacionales, como resultado de los procesos de descentralización y entrega de autonomías. Este fenómeno que respondió a la solución de la crisis del centralismo de la mayoría de los países latinoamericanos, configuró una tendencia contraria a los procesos de reducción y racionalización propuestos inicialmente dentro del modelo neoliberal (Sánchez 1993, Oszlack 2000 y Moncayo 2006).

Además de afectar el funcionamiento interno de los aparatos administrativos del sector público, las dificultades en materia organizacional pueden deformar las relaciones con la sociedad. En ese sentido, la simplificación administrativa debe entenderse como el conjunto de acciones orientadas a analizar, identificar, clasificar y mejorar los procesos administrativos del sector público, buscando la reducción de cargas tanto en materia administrativa para el mejor funcionamiento interno de las entidades públicas, como de trámites para la ciudadanía, las empresas y demás organismos con los que se relacionan cada una de estas entidades.

En esos términos, es posible identificar diferentes manifestaciones de reforma y uso de herramientas de simplificación y racionalización administrativa que han contribuido a alimentar condiciones cada vez más favorables en las dinámicas de relacionamiento Estado-sociedad, entre los diferentes países de regiones tan 
disímiles como Europa, Norteamérica y América Latina.

\section{Propósitos generales de las dinámicas recientes de simplificación y racionalización administrativa}

Entre los desarrollos más importantes que marcan las actuales tendencias de simplificación y racionalización administrativa, es posible identificar, tanto desde la norma como desde la puesta en práctica, casos valiosos que vale la pena resaltar. Dichos casos pueden ubicarse en distintas regiones como Norte América, América Latina y Europa, con contextos institucionales, culturales y económicos muy distintos. Sin embargo, los propósitos generales persiguen logros similares independientemente de los contextos propios de cada país.

Dentro de esa lógica, los casos de Francia, España, Canadá, México y Chile pueden considerarse como casos de éxito factibles de ser tomados en cuenta como punto de referencia en relación con nuestra actual política antitrámites. Las normas que rodean las actuales políticas de estos países frente a este tema se resumen en la siguiente tabla.

Tabla 1. Principales normas que enmarcan la estructura jurídico-política antitrámites en España, Francia, Canadá, México, Chile y Colombia.

\begin{tabular}{|c|c|c|}
\hline PAís & NORMAS PRINCIPALES & NORMAS COMPLEMENTARIAS \\
\hline España & $\begin{array}{l}\text { Ley } 30 \text { de 1992, denominada Régimen Jurí- } \\
\text { dico de las Administraciones Públicas y del } \\
\text { Procedimiento Administrativo Común. } \\
\text { Ley } 11 \text { del } 22 \text { de junio de } 2007 \text { sobre Acce- } \\
\text { so Electrónico de los ciudadanos a los Ser- } \\
\text { vicios Públicos. }\end{array}$ & $\begin{array}{l}\text { Real Decreto 670/1999, del } 23 \text { de abril. } \\
\text { Real Decreto 772/1999, de } 7 \text { de mayo. } \\
\text { Real Decreto 522/2006, de } 28 \text { de abril. } \\
\text { Real Decreto 523/2006, de } 28 \text { de abril. } \\
\text { Real Decreto 887/2006, de } 21 \text { de julio. } \\
\text { Ley } 17 \text { de 2009 (23 de noviembre). } \\
\text { Directiva 2006/123 CE (Consejo Europeo). }\end{array}$ \\
\hline Francia & $\begin{array}{l}\text { Ley No } 2000-321 \text { del } 12 \text { de abril de } 2000 \text { so- } \\
\text { bre los derechos de los ciudadanos en sus } \\
\text { relaciones con el Gobierno. } \\
\text { Decreto No } 2000-1277 \text { del } 26 \text { de diciembre } \\
\text { de } 2000 \text { que elimina documentos y proce- } \\
\text { dimientos que se exigían antes. } \\
\text { Decreto No } 2001-89 \text { del } 1 \text { de octubre de } \\
2001 \text { reglamenta lo relacionado con las co- } \\
\text { pias autenticadas de documentos oficiales. }\end{array}$ & $\begin{array}{l}\text { Ley } 2003-591 \text { de } 2003 \text {. } \\
\text { Ley No 2007-1787 de } 20 \text { de diciembre de } 2007 . \\
\text { Decreto 2003-1099de } 20 \text { noviembre de } 2003 . \\
\text { Decreto } 2005-1792 \text { de } 30 \text { de diciembre de } 2005 . \\
\text { Ordenanza No 2005-1516 de diciembre de } 2005 .\end{array}$ \\
\hline Canadá & $\begin{array}{l}\text { Directiva del Gabinete para la Racionaliza- } \\
\text { ción del Reglamento (CDSR). } \\
\text { Iniciativa para la reducción de carga de trá- } \\
\text { mites (PBRI). }\end{array}$ & Iniciativa para el mejoramiento del servicio en Canadá (1999). \\
\hline México & $\begin{array}{l}\text { Ley Federal de Transparencia y Acceso a la } \\
\text { Información Pública Gubernamental (2002). }\end{array}$ & $\begin{array}{l}\text { Código de Etica de la Administración Federal (2002). } \\
\text { Ley del Servicio Civil de Carrera. } \\
\text { Programa Federal de Combate a la Corrupción. }\end{array}$ \\
\hline
\end{tabular}




\begin{tabular}{|c|c|c|}
\hline PAÍS & NORMAS PRINCIPALES & NORMAS COMPLEMENTARIAS \\
\hline Chile & $\begin{array}{l}\text { Ley No } 19.880 \text { de } 2003 \text { que establece las Ba- } \\
\text { ses de los Procedimientos Administrativos } \\
\text { que rigen los actos de los Órganos de la Ad- } \\
\text { ministración del Estado. } \\
\text { Decretos Supremos en materia de Gobier- } \\
\text { no Electrónico: } 77 \text { de } 2004,81 \text { de } 2004,83 \\
\text { de } 2004,93 \text { de } 2006 \text {, y } 100 \text { de } 2006 .\end{array}$ & $\begin{array}{l}\text { Instructivo Presidencial No } 041 \text { de } 1998 . \\
\text { Instructivo Presidencial No } 04 \text { de } 2003 . \\
\text { Decreto } 475 \text { (reglamentario de la ley 19553). } \\
\text { Ley } 19.553 \text { de } 1998 . \\
\text { Ley } 19.799 \text { de } 2002 . \\
\text { Ley } 20.212 \text { de } 2007 \text {. } \\
\text { Ley } 20.285 \text { de } 2008 . \\
\text { GAB.PRES No } 008 \text { de } 4 \text { de diciembre de } 2006 .\end{array}$ \\
\hline Colombia & 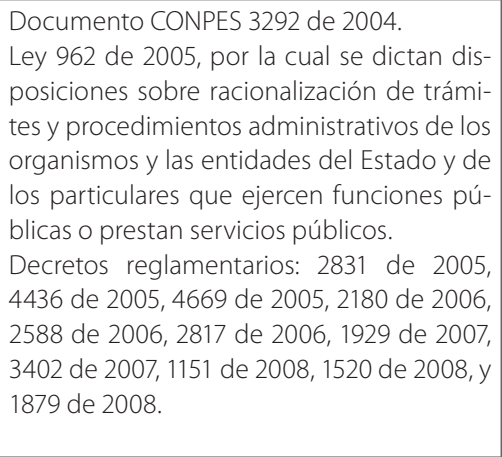 & $\begin{array}{l}\text { Ley } 190 \text { de } 1995 \text { LEstatuto Anticorrupción. } \\
\text { Ley } 87 \text { de } 1993 \text { y decreto } 1575 \text { de 2001, directiva presiden- } \\
\text { cial } 02 \text { de } 1994 \text { y decreto } 1599 \text { de } 2005 \text { (Normas sobre con- } \\
\text { trol interno). } \\
\text { Ley } 200 \text { de } 1995 \text { Estatuto anticorrupción. } \\
\text { Decreto } 2150 \text { de } 1995 . \\
\text { Ley } 489 \text { de } 1998 \text { Estatuto Básico de Organización y Funciona- } \\
\text { miento de la Administración Pública. } \\
\text { Ley } 527 \text { de } 1998 \text { (reglamenta el acceso y uso de los mensajes } \\
\text { de datos, del comercio electrónico y de las firmas digitales) y } \\
\text { Decreto } 1747 \text { de } 2000 . \\
\text { Decreto } 4485 \text { de } 2009 \text { (Por medio de la cual se adopta la nor- } \\
\text { ma técnica de calidad de la Gestión Pública). }\end{array}$ \\
\hline
\end{tabular}

Fuente: elaborada por la autora, con base en los marcos normativos sobre simplificación

de trámites en España, Francia, Canadá, Chile, Brasil y Colombia.

En términos generales, las diferentes reformas sobre reducción de trámites se componen de fenómenos configurados alrededor de principios como la orientación hacia el ciudadano y la eficacia del sector público. En el primer caso, el énfasis se encuentra puesto en el fortalecimiento de la calidad y los niveles de cobertura en materia de bienes y servicios públicos. En el segundo caso, cuando se hace referencia a la forma como opera internamente el sector público, articulado por supuesto a la necesidad de prestar a los ciudadanos unos servicios de manera más eficiente, se requiere corregir las disfuncionalidades en el actuar administrativo público logrando no sólo mejores resultados sino además en el menor tiempo y al menor costo posible.

Esto implica, por un lado, propender por la búsqueda de objetivos vinculados con la calidad de los servidores públicos en relación con los niveles de formación y experiencia que contribuyan no sólo al mejoramiento de la eficiencia y la eficacia, sino también al fortalecimiento de los procesos de transparencia y reducción de corrupción. Dichos procesos, además, deben ser garantizados tanto por la disposición de mecanismos de control, como por el aumento en los niveles de compromiso y ética del servidor público.

Por otro lado, el Estado y su administración pública se encuentran, en la actualidad, sometidos a importantes presiones que provienen del contexto social, económico, político y por supuesto tecnológico (Rodríguez, 2003). Estas presiones derivadas de los procesos de transformación, inherentes a las dinámicas globalizadoras, hacen que el sector público tenga que flexibilizarse con el fin de lograr procesos de adaptación y respuesta más rápidas y coherentes con las exigencias del entorno. 
De acuerdo con estos elementos, las diferentes políticas y procesos de simplificación administrativa y racionalización de trámites a nivel internacional, igual que sucede con la política colombiana, se ubican dentro de la lógica del mejoramiento de las relaciones de los particulares con el Estado. Estas relaciones se encuentran enmarcadas, en primer lugar, en el acceso a bienes y servicios que éste ofrece, derivados del ejercicio de derechos consagrados en la Constitución y la ley y, en segundo lugar, en el cumplimiento de deberes que tienen tanto ciudadanos como empresas frente al Estado. En la medida en que dichos derechos y deberes se materializan en un acercamiento de los particulares con la administración y en la realización de trámites, expresados en inversión de tiempo, recursos y esfuerzos, la política antitrámites se orienta hacia un mejoramiento de las condiciones a las que se tienen que someter ciudadanos y empresas en sus relaciones con el Estado y la administración pública.

De igual manera, y dado que el tema de trámites implica en sentido estricto un momento en una cadena más amplia de procesos y procedimientos internos de la administración pública, las diferentes políticas ubican la racionalización de trámites dentro de la lógica más amplia de la simplificación administrativa. Ésta, implica concebir la racionalización o simplificación en el funcionamiento de la administración en un sentido más complejo que la simple relación directa de la administración con los particulares en la gestión de deberes y derechos, ya que para lograr este último resultado se requiere de la transformación sistemática de todo el engranaje administrativo que determina el funcionamiento del Estado. Esta concepción, que está presente en el contexto internacional, por supuesto, también está presente dentro de la política planteada para Colombia.

Sin embargo, a diferencia de la política antitrámites en Colombia, además del mejoramiento de las relaciones entre los particulares y la administración, las políticas de simplificación administrativa y de trámites de países como Canadá, España y Francia, se enmarcan claramente en objetivos de desarrollo económico. Estos objetivos se encuentran planteados en el caso de Espańa y Francia en la directiva 2006/123 del Consejo Europeo y en el caso de Canadá en orientaciones de la OCDE que encierran el mismo propósito. Dicho propósito implica la reducción de cargas administrativas y de trámites injustificados que generen esfuerzos y costos que afecten la actividad económica especialmente para el caso de las pequeñas y medianas empresas.

Adicionalmente, la simplificación administrativa y de trámites se encuentra articulada para todos los países, con el bobierno electrónico, el cual, más que ser un fin en sí mismo, se constituye en una herramienta que surge como respuesta a las necesidades cambiantes del contexto en el que se desenvuelven los Estados y sus administraciones públicas. Esto, debido a que el uso de las tecnologías de la información se ha vuelto, cada vez más, una herramienta imprescindible en el mejoramiento de las relaciones entre Estado, empresas y particulares.

El e-gobierno sirve a propósitos variados que se encuentran planteados desde la misma concepción de modernización del estado dentro de la lógica de Nueva Gestión Pública. Estos son: eficiencia, eficacia, transparencia, orientación al usuario, confiabilidad y acceso a la información, entre otros. Su importancia 
no sólo se ubica en la dinámica de reducción de trámites sino también en el mejoramiento del funcionamiento interno de la administración pública, de las relaciones entre los diferentes organismos y niveles que conforman el Estado y de la comunicación entre el Estado y la sociedad en general. Es decir que constituye una herramienta muy valiosa en términos de funcionamiento del Estado pero también de legitimidad y fortalecimiento de los niveles de gobernabilidad.

\section{La política de racionalización de trámites en Colombia frente al contexto internacional}

Tomando en cuenta las diferentes normas que rodean los procesos de racionalización y simplificación administrativa de los distintos países tomados como referencia, es posible identificar varias estrategias globales tendientes a mejorar los mecanismos de relacionamiento entre los particulares y la administración pública, con el fin de reducir los requerimientos planteados desde el Estado o de mejorar los mecanismos de acceso a la administración.

En este sentido, fue posible identificar dos categorías claramente diferenciables. Por un lado, las distintas normas se orientan a la definición de directrices que propenden por un mejor trato a los particulares en relación directa con la reducción de los requisitos y cargas en materia de trámites ${ }^{2}$. Por otro lado, los marcos

De acuerdo con la Guía Nacional para la simplificación, el mejoramiento y la racionalización de procesos, procedimientos y normativos definen propósitos cuya lógica se encuentra orientada hacia el funcionamiento interno de la administración. Ambas categorías resultan ser necesarias dentro de los procesos de simplificación, ya que no es suficiente el ajuste de los instrumentos que permiten el contacto directo entre usuarios y administración. Se requiere, además, considerar este fenómeno como un momento de la administración dentro de una cadena más amplia de procesos y procedimientos administrativos. Incluyendo, claro está, aquellas dinámicas que tocan el funcionamiento interno de los organismos y entidades públicas aunque no concluyan en un momento de contacto directo con la sociedad, por efectos de trámites.

\section{a. Medidas que involucran el contacto directo con los particulares}

Los distintos desarrollos jurídicos de los países consultados incluyen dentro de sus propósitos, directrices que persiguen la reducción de los requisitos exigidos a los particulares. Adicionalmente, algunas de estas normas incluyen herramientas relacionadas con la protección del ciudadano y el mejoramiento permanente del servicio, con miras al cubrimiento de las expectativas que surgen desde la sociedad en materia de trámites. En el primer caso, se trata de la eliminación de exigencias innecesarias en los siguientes términos:

trámites (2009) del Departamento Administrativo de la Función Pública de Colombia, se debe considerar como trámite al... conjunto, serie de pasos o acciones reguladas por el Estado, que deben efectuar los usuarios para adquirir un derecho o cumplir con una obligación prevista o autorizada por la ley. 
- No aportación de copias de los documentos presentados.

- Eliminación de las copias autenticadas.

- Prohibición de declaraciones extrajuicio.

- No dirigir el mismo escrito a más de un Órgano.

- No aportación de documentos que obran en poder de la administración: esta iniciativa se encuentra planteada en las iniciativas de España, Chile y Brasil.

- No exigibilidad de certificados o documentos expedidos por otros organismos.

- Prohibición de solicitud de documentos competencia de otras autoridades.

- Prohibición de exigencia de autorizaciones, requisitos o permisos que no estén previstos en la ley.

Además de la reducción de requisitos, las distintas normas introducen herramientas que permiten, o por lo menos buscan, la medición permanente de la satisfacción de los ciudadanos frente a la implementación de estas medidas. Entre estas alternativas se cuentan:

- Encuestas de percepción ciudadana sobre los servicios que ofrece la administración pública: esta herramienta ha recibido especial importancia en Canadá y se enmarca en la Iniciativa para el mejoramiento del servicio, con el fin de mejorar los niveles de satisfacción de los clientes a los servicios del Estado. Adicionalmente la Iniciativa para reducir la carga de trámites enfocada hacia las pequeñas y medianas empresas mide permanentemente el impacto de la carga de trámites que se generan por efecto de las regulaciones, a través de la Encuesta de Cumplimiento Normativo de Costos.

- Revisión permanente de requisitos exigidos a pequeñas y medianas empresas: además de las consultas que se realizan a través de la encuesta mencionada, en Canadá existe un Comité Consultivo para la Pequeńa empresa y el espíritu empresarial (ACSBE). Este comité de origen privado, se creó para aconsejar al gobierno sobre cómo mejorar aún más el acceso empresarial a los programas federales y la información.

- Simplificación del lenguaje administrativo: esta iniciativa, que es de origen francés, depende de la existencia de un Comité Directivo para la Simplificación del Lenguaje Administrativo (COLSA). Dicho comité, que se encuentra compuesto por personalidades del mundo y voluntarios y expertos lingüistas, se encarga de formular propuestas para mejorar la calidad del lenguaje administrativo de documentos y formularios y supervisar su aplicación por el Gobierno.

- Simplificación Administrativa: Francia cuenta también con un comité Directivo para la Simplificación Administrativa, responsable de asesorar la proyección de leyes, ordenanzas y decretos con el fin de simplificar y aclarar el sistema jurídico vigente. Por su parte, Colombia cuenta con el Grupo de Racionalización y Automatización de Trámites (GRAT), y con el DAFP en el proceso de aprobación de nuevos trámites.

- Instancias de protección de los derechos de los ciudadanos: además de lo anterior, el marco institucional francés ha creado una serie de instancias cuyo propósito es mejorar las relaciones 
entre el ciudadano y la administración, buscando proteger principalmente al primero. Entre las instancias disponibles para esta labor encontramos el defensor del pueblo, la Comisión Nacional de Informática y de las Libertades (CNIL) y la Comisión de Acceso a Documentos Administrativos (CADA).

\section{b. Medidas vinculadas con las actuaciones de la administración pública}

En relación con este conjunto de medidas, también es posible identificar dos grupos distintos de herramientas. En primer lugar, están aquellas que pueden afectar los procedimientos internos de la administración pública en materia de trámites y en segundo lugar, es posible identificar conjuntos de herramientas vinculadas con el uso de medios electrónicos, dentro de la tendencia de gobierno electrónico. En el primer caso, las medidas identificadas incluyen las siguientes alternativas:

- Carta de servicios al ciudadano: esta propuesta, que hace parte de la política española, busca informar a los ciudadanos de los servicios prestados por la dependencia o entidad, las formas de acceso a estos servicios y su compromiso con los estándares de calidad de servicio al cliente.

- Gratuidad de los actos necesarios para el ejercicio de la ciudadanía

- Establecimiento de modelos normalizados de solicitudes: formularios, guías y otros documentos, así como para su utilización.

- Derogación de normas ilegales o irrelevantes: a este respecto la política francesa plantea como posibilidad que una autoridad, de oficio o a petición de una persona interesada, derogue las normas expresamente ilegales o irrelevantes.

- Recibo de documentos (uso o no de sellos de recibo): con respecto a este punto existen divergencias en materia de la pertinencia o no del uso de los sellos de recibo en la realización de trámites. En el caso colombiano estos están prohibidos, sin embargo, la política francesa los considera como una necesidad y en el caso de España se habla de registro electrónico.

- Remisión de documentos a la autoridad competente: en la política francesa se encuentra establecido que cuando un ciudadano dirija alguna solicitud a la autoridad equivocada, ésta debe remitirla a la autoridad competente y notificar al interesado. En todos los casos se emite recibo por la autoridad competente.

- Cumplimiento de los plazos de acuerdo con el procedimiento y el acto que genera: a este respecto, la política chilena introduce plazos específicos vinculados con procedimientos de trámites. De igual manera, se mencionan tanto el silencio negativo como el positivo en relación con el vencimiento de los plazos relacionados con trámites:

- Silencio administrativo negativo: en este caso, la política chilena plantea que se debe entender como rechazada una solicitud que no se resuelva dentro del plazo legal cuando ella afecte el patrimonio fiscal. El caso español, por su parte, a través de la ley 30 de 1992, estableció (artículo 43.3) el silencio administrativo negativo en dos casos 1) procedimientos de ejercicio del derecho de petición del artículo 29 de la Constitución Española y 2) la resolución 
de los recursos administrativos. Adicionalmente, la política francesa considera dentro del silencio administrativo únicamente la opción del silencio negativo, el cual se configura cuando una autoridad guarda silencio durante más de dos meses, lo cual es equivalente a un rechazo.

- Silencio administrativo positivo: se configura luego de transcurrido el plazo legal para resolver acerca de una solicitud y sin que la administración se pronuncie sobre ella. En este caso, el interesado puede denunciar el incumplimiento del plazo ante la autoridad competente. Esta figura está considerada en las políticas de Chile y España.

- Expediente escrito.

- Notificación escrita.

- Derecho de turno para peticiones, quejas y reclamos: igual que los demás instrumentos que aparecen a continuación en este apartado, éste corresponde a la política colombiana vigente.

- Centros de servicio al ciudadano y despachos públicos: este conjunto de medidas que también está presente en la normatividad colombiana incluye las siguientes herramientas:

- Despacho a los usuarios que hayan ingresado dentro de las 8 horas de atención al público.

- Prohibición de la retención de documentos para el ingreso a organismos públicos o privados.

- Uso de medios electrónicos para asignación de citas o turnos de atención.

Por otro lado, en relación con el uso de los medios electrónicos disponibles y la implementación de nuevas tecnologías, la política española (Fundación Orange, 2010) ofrece una importante relación de medidas que articulan los propósitos de simplificación con el e-gobierno. Estas medidas incluyen:

- Igualdad y accesibilidad a los servicios: está determinada por los canales y medios disponibles con los que cuenta un ciudadano para poder realizar un servicio o trámite a las administraciones, independientemente de sus limitaciones físicas, edad o conocimiento de los medios tecnológicos.

- Canales de acceso a los servicios: hace referencia a la incorporación de nuevas tecnologías para el funcionamiento interno de la administración. También hace referencia a la posibilidad de que los ciudadanos que no puedan acceder electrónicamente a la administración pública dispongan de medios adecuados para comunicarse manteniendo los mismos derechos y garantías. Entre los canales de acceso a los servicios se encuentran el internet, el teléfono, el correo físico y los centros de atención presencial entre otros.

- Expediente electrónico: se refiere al conjunto de documentos electrónicos correspondientes a un procedimiento administrativo.

- Acceso electrónico al estado de tramitación de expedientes: la posibilidad de que el ciudadano pueda consultar el estado de un trámite iniciado electrónicamente. Al respecto los ciudadanos pueden acceder utilizando el certificado digital, el número de expediente o código de procedimiento, o alguna clave identificadora provista al inicio de la tramitación.

- Interoperabilidad: incluye la interoperabilidad técnica, semántica y 
organizativa de los sistemas y aplicaciones empleados por las administraciones públicas. Cada administración debe facilitar el acceso de las restantes administraciones públicas a los datos relativos a los interesados que obren en su poder y se encuentren en soporte electrónico.

- Seguridad jurídica: la finalidad es generar confianza en el uso de los medios electrónicos a través de normas para garantizar la seguridad de los sistemas, los datos, las comunicaciones y los servicios electrónicos de tal forma que permitan el ejercicio de derechos y el cumplimiento de deberes a través de estos medios.

- Ventanillas únicas: se refiere a la posibilidad de realizar trámites a través de ventanilla única, por vía electrónica y a distancia.

- Documento administrativo electrónico: es toda representación de un hecho, con datos o imágenes, que está archivado en un soporte electrónico, que es susceptible de identificación y tratamiento diferenciado y el cual ha de estar firmado electrónicamente. A este respecto se incluye la digitalización de los documentos privados aportados por los ciudadanos y el uso del sello electrónico. También se debe disponer de un portal donde se pueda comprobar la autenticidad de un documento en papel o de un documento emitido por la administración.

- Copia electrónica de documentos: tiene relación tanto con las realizadas a partir de documentos emitidos originariamente en papel, como de las copias de documentos que ya estuvieran en soporte electrónico y las condiciones para realizar en soporte papel co- pia de originales emitidos por medios electrónicos, o viceversa.

- Firma electrónica: constituye un medio de identificación electrónica necesario para que los ciudadanos puedan establecer y validar sus relaciones con las administraciones públicas. Los sistemas de firma electrónica más utilizados son el eDIN y los emitidos por la Fábrica Nacional de la moneda y de Timbre (FNMT), la AC Camefirma (de la Cámara de Comercio) y la ACA (Autoridad de Certificación de la Abogacía) y la firma profesional.

- Firma electrónica avanzada: este mecanismo se encuentra planteado en algunos casos como Chile donde se impone como obligatoria sólo para aquellos documentos que revisten naturaleza de instrumento público o que se desea produzcan efectos jurídicos (Comunidad tecnológica gubernamental de Chile, 2010).

- Sede administrativa electrónica: es la clave de la comunicación jurídica con los ciudadanos en soporte electrónico.

- Estándares abiertos: se refiere a la neutralidad tecnológica a través del uso de software que sea público y su utilización esté disponible de forma gratuita o a un costo que no suponga dificultades generales de acceso. Además, su uso y aplicación no debe estar condicionado al pago de un derecho de propiedad intelectual o industrial.

- Registro electrónico: consiste en constatar la fecha de entrada de un documento, anotar el asiento de la entrada/ salida, guardar los datos de la presentación de información y devolver un acuse de recibo con el número de registro y momento de la presentación. El registro puede incluir además, el sellado de tiempo, el cotejo/compulsa 
electrónica de documentos presentados físicamente o el funcionamiento como registro único para toda la administración. Adicionalmente, incluye la emisión automática de un recibo que es una copia autenticada del escrito, solicitud o comunicación, incluyendo fecha y hora de presentación y el número de entrada de registro.

- Notificación electrónica: notificación por medios electrónicos, como SMS, correo electrónico, teléfono móvil o mediante el Sistema Único de Notificaciones Electrónicas Seguras, a través del cual las notificaciones llegan a la bandeja de notificaciones de la dirección Electrónica Única del Ministerio de Política Territorial. Este servicio puede ser solicitado de manera voluntaria y gratuita por cualquier persona y funciona mediante una dirección electrónica.

- Disponibilidad de un catálogo online de servicios electrónicos: las comunidades autónomas deben publicar en sus webs, catálogos sobre los servicios electrónicos que ofrecen sus respectivas administraciones.

- Sistematización de trámites: España ha implementado, para este propósito, herramientas como el Sistema de Información Administrativa (SIA) y el Sistema de Evaluación de Cargas que incluye los índices (IEB) y (SCM). En Colombia encontramos el SUIT en ese mismo sentido.

A continuación se presenta una síntesis de las distintas medidas descritas:

Tabla 2. Síntesis de la estructura jurídico política antitrámites de Canadá, España, Francia, Chile, México y Colombia.

\begin{tabular}{|c|c|c|}
\hline DIMENSIONES & SUB-DIMENSIONES & $\begin{array}{c}\text { INSTRUMENTOS CONSIDERADOS EN LAS POLÍTICAS } \\
\text { ANTITRÁMITES ESTUDIADAS }\end{array}$ \\
\hline \multirow[t]{2}{*}{$\begin{array}{l}\text { EN RELACIÓN CON LOS } \\
\text { PARTICULARES }\end{array}$} & $\begin{array}{l}\text { Sobre los requisitos } \\
\text { exigidos a los } \\
\text { particulares }\end{array}$ & $\begin{array}{l}\text { No aportación de copias de los documentos presentados. } \\
\text { No dirigir el mismo escrito a más de un Órgano. } \\
\text { No aportación de documentos que obran en poder de la Admi- } \\
\text { nistración. } \\
\text { No exigibilidad de certificados o documentos expedidos por otros } \\
\text { organismos. } \\
\text { Prohibición de declaraciones extrajuicio.* } \\
\text { Prohibición de solicitud de documentos competencia de otras au- } \\
\underline{\text { toridades. }} \\
\text { Prohibición de exigencia de autorizaciones, requisitos o permisos } \\
\text { que no estén previstos en la ley. } \\
\text { Eliminación de las copias autenticadas. }\end{array}$ \\
\hline & $\begin{array}{l}\text { Sobre el mejoramiento } \\
\text { permanente del } \\
\text { servicio al ciudadano }\end{array}$ & $\begin{array}{l}\text { Encuestas de percepción ciudadana sobre los servicios que ofrece } \\
\text { la administración pública. } \\
\text { Revisión permanente de requisitos exigidos a pequeñas y media- } \\
\text { nas empresas. } \\
\text { Simplificación del lenguaje administrativo. } \\
\text { Instancias de protección de los derechos de los ciudadanos. }\end{array}$ \\
\hline
\end{tabular}




\begin{tabular}{|c|c|c|}
\hline DIMENSIONES & SUB-DIMENSIONES & $\begin{array}{c}\text { INSTRUMENTOS CONSIDERADOS EN LAS POLÍTICAS } \\
\text { ANTITRÁMITES ESTUDIADAS }\end{array}$ \\
\hline \multirow{2}{*}{$\begin{array}{l}\text { EN RELACIÓN CON } \\
\text { LA ADMINISTRACIÓN } \\
\text { PÚBLICA }\end{array}$} & $\begin{array}{l}\text { Sobre los } \\
\text { procedimientos de la } \\
\text { administración pública } \\
\text { en materia de trámites }\end{array}$ & $\begin{array}{l}\text { Carta de Servicios al Ciudadano. } \\
\text { Establecimiento de modelos normalizados de solicitudes: formula- } \\
\text { rios, guías y otros documentos, así como para su utilización. } \\
\text { Derogación de normas ilegales o irrelevantes. } \\
\text { Remisión de documentos a la autoridad competente. } \\
\text { Silencio Administrativo positivo. } \\
\text { Silencio Administrativo negativo. } \\
\text { Expediente escrito. } \\
\text { Notificación escrita. } \\
\text { Gratuidad de los actos necesarios para el ejercicio de la ciudadanía } \\
\text { Recibo de documentos (uso o no de sellos de recibo). } \\
\text { Respetar el derecho de turno para peticiones, quejas y reclamos. } \\
\text { Despacho a los usuarios que hayan ingresado dentro de las } 8 \text { ho- } \\
\text { ras de atención al público. } \\
\text { Prohibición de la retención de documentos para el ingreso a orga- } \\
\text { nismos públicos o privados. } \\
\text { Uso de medios electrónicos para asignación de citas o turnos de } \\
\text { atención. }\end{array}$ \\
\hline & $\begin{array}{l}\text { Uso de los medios } \\
\text { electrónicos } \\
\text { disponibles e } \\
\text { implementación de } \\
\text { nuevas tecnologías }\end{array}$ & $\begin{array}{l}\text { Igualdad y accesibilidad a los servicios. } \\
\text { Canales de acceso a los servicios. } \\
\text { Expediente electrónico. } \\
\text { Acceso electrónico al estado de tramitación de expedientes. } \\
\text { Interoperabilidad. } \\
\text { Seguridad jurídica. } \\
\text { Ventanillas únicas. } \\
\text { Documento administrativo electrónico. } \\
\text { Copia electrónica de documentos. } \\
\text { Firma electrónica. } \\
\text { Firma electrónica avanzada. } \\
\text { Sede administrativa electrónica. } \\
\text { Estándares abiertos. } \\
\text { Registro electrónico. } \\
\text { Notificación electrónica. } \\
\text { Disponibilidad de un catálogo online de servicios electrónicos. } \\
\text { Sistematización de trámites. }\end{array}$ \\
\hline
\end{tabular}

Fuente: Elaborada por la autora, con base en los marcos normativos sobre simplificación

de trámites en España, Francia, Canadá, Chile, Brasil y Colombia.

* Los instrumentos que se encuentran subrayados corresponden a los contenidos

de la actual política antitrámites de Colombia (ley 962 de 2005).

Tomando en cuenta las diferentes informaciones recabadas como producto de la revisión normativa del grupo de países analizados, encontramos que todavía los desarrollos en Colombia en materia de racionalización de trámites son bastante incipientes y básicos. Si bien, como incluye medidas como la eliminación de la exigencia de documentos como las declaraciones extrajuicio, documentos que no estén previstos en la ley o que sean competencia de otras autoridades, todas estas iniciativas todavía están fundadas en la atención presencial, especialmente aquellas que se refieren a la asignación 
de citas o la prohibición de retener documentos a los particulares para ingresar a los organismos públicos o privados.

En estos términos, por lo menos desde la norma, Colombia no cuenta con propósitos claros tendientes a la sistematización plena con énfasis en todos los casos en el uso de medios electrónicos para la racionalización de trámites. A pesar de esto, de acuerdo con los resultados del Informe 2010 sobre Desarrollo de Gobierno Electrónico de la Organización de las Naciones Unidas, Colombia ocupa la posición No. 1 en materia de Gobierno Electrónico en América Latina, pasando del puesto 52 al puesto 31 en el ranking mundial, seguido de Chile que se encuentra en la posición 34 . Este resultado es significativo en la medida en que nos revela que en la práctica los desarrollos en materia de simplificación administrativa y racionalización de trámites han ido en crecimiento, logrando la realización plena de alrededor de 700 trámites completamente en línea (Gobierno en línea, 2010).

De igual manera, gobierno en línea en Colombia ha logrado resultados interesantes en materia de información sobre las distintas entidades públicas y los distintos servicios que prestan, así como coberturas importantes en relación con información sobre organismos del nivel territorial. Por otro lado, aunque estos avances son significativos, Colombia todavía adolece de mecanismos de interlocución con los usuarios de los servicios, que sí están presentes en países europeos y en Norteamérica. Estos mecanismos incluyen, como en el caso de España y Canadá, no sólo consultas a los usuarios sobre el nivel de satisfacción en relación con trámites, sino que además involucran herramientas que promueven la participación de los ciudadanos en los procesos de toma de decisiones (ONU, 2010).

De igual manera, la política antitrámites en Colombia tampoco considera medidas orientadas al uso de medios que faciliten la interoperabilidad, que permitan no sólo el intercambio de información entre entidades, como ya sucede con gobierno en línea, sino que, además, contribuyan a la reducción de documentación exigida al usuario y la disminución de tiempos y costos. Aunque esta alternativa no se encuentra planteada desde la norma, igual que sucede con el caso anterior, Colombia presenta algunos desarrollos en la práctica. Entre estos encontramos la existencia de las denominadas Ventanillas Únicas cuyo propósito es lograr la realización de trámites que involucran varias entidades desde un único despacho o portal Web. Al respecto se pueden mencionar la Ventanilla Única de Comercio Exterior VUCE, la Ventanilla Única para creación de empresas y la Ventanilla Única de Registro a la propiedad, VUR, entre otras.

Finalmente, frente a la posibilidad de aprovechar las alternativas que ofrecen otros países, Colombia tendría una gran oferta de estrategias y medidas bastante pertinentes para ser aplicadas en nuestro contexto. En especial, se debe resaltar la experiencia francesa y canadiense en materia de mecanismos que consultan la opinión ciudadana y que garantizan que el funcionamiento del Estado se ajuste a las necesidades y preferencias de sus ciudadanos y empresas. Francia se convierte en un caso ejemplarizante en la medida que muchas de sus herramientas se relacionan con mecanismos de protección de los usuarios frente al Estado, especialmente en relación con ciertas 
instancias mediadoras en casos de conflicto. Adicionalmente, resulta determinante el caso español donde la norma, si bien es reciente, articula propósitos claros de racionalización con el uso de las tecnologías de la información.

\section{Conclusiones}

La simplificación administrativa y la racionalización de trámites son tendencias que se ubican dentro de las nuevas dinámicas de reforma estatal cuyo marco fundamental es el paradigma de la Nueva Gestión Pública. Este paradigma, que acompañó la configuración de la administración pública correspondiente al Estado neoliberal, tiene como propósito intermedio estimular la adopción de instrumentos y herramientas que han marcado el éxito de la gestión del sector privado. Entre los principios rectores de dicho paradigma encontramos la eficiencia, eficacia, economía, calidad, transparencia y orientación al cliente, entre otros.

Debido a los vicios derivados de la aplicación del modelo weberiano en el sector público, se generaron dinámicas de relacionamiento negativas con la sociedad, de tal manera que entre los nuevos objetivos vinculados con el cambio de modelo de administración pública se encontraba el de mejorar las relaciones con los ciudadanos. Con lo anterior se buscó, en términos generales, reducir requisitos, tiempos y costos en la realización de los trámites en aquellas circunstancias en las que, por marco legal, los ciudadanos y las empresas deben acercarse a la administración bien sea para el acceso a servicios o para el cumplimiento de obligaciones.

En términos generales, las políticas públicas de racionalización de trámites giran alrededor de herramientas que se pueden agrupar en dos categorías generales. En primer lugar, encontramos medidas vinculadas con los particulares en relación con los requisitos que se les exige cuando deben realizar un trámite y también, con los instrumentos en favor del ciudadano para el mejoramiento continuo del servicio. En segundo lugar, encontramos medidas relacionadas con la actuación de la administración pública en su funcionamiento interno y el uso de instrumentos tecnológicos para garantizar la eficiencia y eficacia en la agilización de los trámites que realizan ciudadanos y empresas.

Al comparar la política colombiana con los desarrollos logrados por otros países, se hace evidente que nuestro marco institucional es todavía incipiente en racionalización de trámites, especialmente en lo que toca a la consideración del uso de medios electrónicos y la eliminación paulatina de la atención presencial. Por otro lado, se requiere prestar más atención a los posibles mecanismos de interoperabilidad que permitan que los distintos organismos y entidades del Estado interactúen y compartan información, con el fin de facilitar por un lado la gestión pública y por otro, la realización de los trámites por parte de los usuarios.

Asimismo, Colombia adolece de mecanismos de interlocución con la sociedad no sólo para la medición de percepción ciudadana sino también en la apertura de canales alternativos de participación y por ende de fortalecimiento democrático. Finalmente, a diferencia de lo que se esperaría, en Colombia la norma no va más allá de la práctica. Por el contrario, es evidente que la experiencia demuestra un mayor desarrollo que la misma norma, lo cual exige un ajuste normativo que vaya a la par con las 
características de nuestro contexto propio y que además plantee nuevas alternativas de fortalecimiento institucional.

\section{Referencias}

I. Bañón, R. y Carrillo, E. (2007). La nueva Administración Pública. Madrid: Alianza Universidad.

2. Córdoba Guerrero, I (2008). Modernización de la administración Pública: la reingeniería de gobierno en Veracruz. Diciembre 13 de 2010. En: http://portal.veracruz.gob.mx/pls/portal/docs/page/colver/difusion/revista_ conciencia/revistano. $9 / 5$.-izcoal $\% 20$ c\%d3rdoba\%20guerrero.pdf

3. Comunidad tecnológica gubernamental de Chile (2010). Firma electrónica. En: http://www.comunidadtecnologica.gob.cl/firma-electronica/

4. DNP (2009). Guía Nacional para la simplificación, el mejoramiento y la racionalización de procesos, procedimientos y trámites.

5. Fundación Orange. (2010). Aplicación de la ley 11 de 2007 de Acceso Electrónico de los Ciudadanos a los Servicios Públicos online en las CC AA españolas.

6. García Sánchez, IM (2007). La Nueva Gestión Pública: evolución y tendencias. Presupuesto y Gasto Púbico 47: 3764. Secretaría General de Presupuestos y Gastos. España.

7. Gobierno en línea (2010). El Gobierno en Línea en Colombia, Situación actual y prospectiva. III Simposio "Las sociedades ante el reto digital" Universidad del Norte. Barranquilla, 17 de marzo de 2010. Diciembre 9 de 2010.
En: http://www.slideshare.net/esaidh/ el-gobierno-en-lnea-en-colombia

8. Guerrero, O. (1998). El "Management" público: una torre de babel. Universidad Nacional Autónoma de México. En: http://omarguerrero.org/articulos/ ManageTorreB.pdf

9. Guijarro, L. (2009). Semantic Interoperability in eGovernment Initiatives. Universidad Politécnica de Valencia, España. En: http://personales.upv. es/lguijar/pubs/EGOV11fGuijarro-SelfArchive.pdf

ıo.Hernández, A. (2007). El espacio de la política en la Gestión Pública. El reto de construir gerencia social orientada por valores públicos, democratizar la administración pública y fortalecer la ciudadanía activa y la acción colectiva. Universidad de los Andes.

I r.López, A. (2003). La Nueva Gestión Pública: algunas Precisiones para su Abordaje Conceptual. Serie I: Desarrollo Institucional y Reforma del Estado Documento No 68. Instituto Nacional de la Administración Pública INAP, Dirección de Estudios e Información.

I 2.Lozada Trabada, A. (2008). Democracia, clientes y calidad total en la administración pública. Revista de Investigaciones Politicas y Sociológicas, año/vol. 7 , número 002. Universidad de Santiago de Compostela, España. P. 39-62.

I 3. Moncayo, E. (2007). La transformación del Estado en América Latina: una perspectiva económica desde los países andinos. Revista Ópera, abril, año/vol. 6, número 006.

I 4. Naciones Unidas (2010). La transformación de los servicios centrados en la ciudadanía. Octubre 11 de 2010. En: http:// unpan1.un.org/intradoc/groups/public/ documents/un/unpan012634.pdf 
I 5. OCDE (2009). Foro sobre la administración tributaria. Subgrupo de servicios a los contribuyentes. Encuesta sobre tendencia y evolución del uso de servicios electrónicos para la prestación de servicios a los contribuyentes.

I6. Oszlack, O. (2000) El mito del Estado mínimo: una década de reforma estatal en Argentina. V Congreso Internacional del CLAD sobre la Reforma del estado y de la Administración Pública, Santo Domingo, Rep. Dominicana 2417 de octubre de 2000. En:

I7.http://www.unpan1.un.org/intradoc/ groups/public/documents/CLAD/ clad0038429.pdf

I 8. Patińo, C. A. (2007). Interoperabilidad. Experienciadee-gobierno en México.Unidad de Gobierno Electrónico y Política de TI Secretaría de la Función Pública. En: http://webcache.googleusercontent.com/ search?q=cache:9gGiUtqSatkJ:www.gobiernofacil.go.cr/e-gob/gobiernodigital/ eventos/presentacionesGEALC/Interop_ Mx.pdf+interoperabilidad+mexico+gobie rno\&cd $=3 \& \mathrm{hl}=\mathrm{es} \& \mathrm{ct}=\mathrm{clnk} \& \mathrm{gl}=\mathrm{co}$

I 9. Ramírez, M.F. (2009, enero-junio). Las Reformas del Estado y la administración pública en América Latina y los intentos de aplicación del New Public Management. Estudios Políticos, 34, Instituto de Estudios Políticos, Universidad de Antioquia, (P. 115-41).

20. Richards, S. (1994). El paradigma del cliente en la gestión pública. Gestión y Análisis de Politicas Públicas, No 1, INAP, Madrid, Septiembre-Diciembre.

2 I. Rodríguez, J. y Tapia, E. (2008). Pensamiento Administrativo y Organizaciones Públicas I. Programa de Administración Pública Territorial. ESAP. Colombia

22. Rodríguez Arana, J. (2002). El proceso de modernización administrativa en las www.rodriguezarana.com/pdf/4_5.pdf Septiembre de 2010.

23.Sánchez Morón, M. (1993). Racionalización Amdinistrativa y Organización Territorial. Revista Española de Derecho Constitucional, Año 14. Num. 40. Enero-Abril 1994.

24. Tornos Mas, J. (2000). La simplificación procedimental en el ordenamiento español. Revista de Administración Pública, No 151, Enero-abril 2000. España.

\section{Normas \\ España}

I. Real Decreto 670/1999, del 23 de abril

2. Real Decreto $772 / 1999$, de 7 de mayo.

3. Real Decreto 522/2006, de 28 de abril.

4. Real Decreto 523/2006, de 28 de abril.

5. Real Decreto 887/2006, de 21 de julio.

6. Ley 30 de 1992.

7. Ley 38 de 2003 (17 de noviembre).

8. Ley 11 de 2007 (22 de junio).

9. Ley 17 de 2009 (23 de noviembre).

Io.Directiva 2006/123 CE (Consejo Europeo).

\section{Francia}

I. Ley No 2000-321 del 12 de abril de 2000.

2. Ley 2003-591 de 2003

3. Ley No 2007-1787 de 20 de diciembre de 2007.

4. Decreto No 2000-1277 del 26 de diciembre de 2000.

5. Decreto No 2001-89 del 1 de octubre de 2000.

6. Decreto 2003-1099de 20 noviembre de 2003.

7. Decreto 2005-1792 de 30 de diciembre de 2005 .

8. Ordenanza No 2005-1516 de diciembre de 2005 . 


\section{Canadá}

I. Iniciativa para el mejoramiento del servicio en Canadá (1999).

2. Directiva del Gabinete para la Racionalización del Reglamento (CDSR) (2007).

3. Iniciativa para la reducción de carga de trámites (PBRI) (2004).

\section{Chile}

I. Instructivo Presidencial No 041 de 1998.

2. Instructivo Presidencial No 04 de 2003.

3. Decreto 475 (reglamentario de la ley 19.553).

4. Ley 19.553 de 1998.

5. Ley 19.799 de 2002.

6. Ley No 19.880 de 2003.

7. Ley 20.212 de 2007.

8. Ley 20.285 de 2008.

9. GAB.PRES No 008 de 4 de diciembre de 2006

Io.Decreto Supremo No 77 de 2004.

I I. Decreto Supremo No 81 de 2004.

I 2. Decreto Supremo No 83 de 2004.

I 3. Decreto Supremo No 93 de 2006.

I 4. Decreto Supremo No 100 de 2006.

\section{México}

I. Ley Federal de Transparencia y Acceso a la Información Pública Gubernamental (2002).
2. Código de Etica de la Administración Federal (2002).

3. Ley del Servicio Civil de Carrera.

4. Programa Federal de Combate a la Corrupción.

\section{Colombia}

I. Documento CONPES 3292 de 2004.

2. Ley 962 de 2005, por la cual se dictan disposiciones sobre racionalización de trámites y procedimientos administrativos de los organismos y las entidades del Estado y de los particulares que ejercen funciones públicas o prestan servicios públicos.

3. Decretos reglamentarios 4669 de 2005 y 1151 de 2008.

4. Ley 190 de 1995 Estatuto Anticorrupción.

5. Ley 87 de 1993 y decreto 1575 de 2001, directiva presidencial 02 de 1994 y decreto 1599 de 2005 (Normas sobre control interno).

6. Ley 200 de 1995 Estatuto Anticorrupción.

7. Decreto 2150 de 1995.

8. Ley 489 de 1998 Estatuto Básico de Organización y Funcionamiento de la Administración Pública.

9. ley 527 de 1998 (reglamenta el acceso y uso de los mensajes de datos, del comercio electrónico y de las firmas digitales) y Decreto 1747 de 2000. 